\title{
Unusually short excited state lifetimes of indenofluorene and fluorenofluorene derivatives result from a conical intersection
}

\author{
Bradley D. Rose ${ }^{a}$, Leah E. Shoer ${ }^{\mathrm{b}}$, Michael R. Wasielewski ${ }^{\mathrm{b}}$, Michael M. Haley ${ }^{\mathrm{a}, *}$ \\ a Department of Chemistry \& Biochemistry and the Materials Science Institute, 1253 University of Oregon, Eugene, OR 97403-1253, United States \\ ${ }^{\mathrm{b}}$ Department of Chemistry and Argonne-Northwestern Solar Energy Research (ANSER) Center, Northwestern University, 2145 Sheridan Road, Evanston, IL \\ 60208-3113, United States
}

\section{A R T I C L E I N F O}

\section{Article history:}

Received 27 August 2014

In final form 14 October 2014

Available online 22 October 2014

\begin{abstract}
A B S T R A C T
The ultrafast excited state decay rates for indenofluorene and fluorenofluorene derivatives as measured by transient absorption spectroscopy are reported. The excited state lifetimes of the molecules were extremely short (ca. 9-12 ps) and are a result of the $p$-xylylene motif in indenofluorenes and the corresponding expanded 2,6-naphthoquinonedimethide in fluorenofluorene. Quantum chemical calculations indicate that the fast relaxation to the ground state results from a potential energy surface crossing between the $S_{0}$ and $S_{1}$ states. This process in turn provides an efficient route for excited state deactivation and explains why this class of quinoidal molecules is non-emissive.
\end{abstract}

(c) 2014 Elsevier B.V. All rights reserved.

\section{Introduction}

There has been much interest in materials for easily produced organic electronics [1]. Applications such as organic photovoltaics (OPVs) and field-effect transistors (OFETs) often require not only high efficiencies but also additional characteristics including processability, flexibility and photoactivity [2-4]. Numerous small-molecule systems have been developed toward this goal that employ extended pi-conjugated centers [5-9]. One such molecular class of interest is the $p$-quinodimethane-containing indenofluorenes and related molecules (e.g., 1-3, Figure 1), which have recently been realized through synthesis [10-17]. Intriguingly, these compounds have been found to be non-emissive and thus we wished to examine their photophysics. One possible mechanism for non-emissive relaxation from a photoexcited state to the ground state is singlet fission, proposed to be present in the expanded system fluorenofluorene (1), due to ideal state energies for singlet fission [18]. This process is particularly desirable in OPVs as it may boost the maximum power conversion efficiency and impede loss pathways from charge recombination $[19,20]$. Another plausible mechanism for the lack of emission is internal conversion through

\footnotetext{
* Corresponding author.

E-mail addresses: m-wasielewski@northwestern.edu (M.R. Wasielewski), haley@uoregon.edu (M.M. Haley).
}

a conical intersection between the ground state, $S_{0}$, and the singlet first excited state, $S_{1}$. Such a mechanism is the cause for efficient internal conversion in $\mathbf{4}$, a substructure of $\mathbf{2}$ [21].

\section{Experimental}

\subsection{Materials}

All molecules were synthesized as previously reported $[10,11,13]$.

\subsection{Optical spectroscopy}

Femtosecond transient absorption (fsTA) measurements were made using a regeneratively amplified Ti:sapphire laser system operating at $832 \mathrm{~nm}$ and a $1 \mathrm{kHz}$ repetition rate as described previously [22-24]. Samples had an absorbance of 0.3-0.7 in dry toluene at the excitation wavelength of $495 \mathrm{~nm}$ and were irradiated in $2 \mathrm{~mm}$ quartz cuvettes with $1 \mu \mathrm{J} /$ pulse focused to a $0.2 \mathrm{~mm}$ diameter spot. The total instrument response function (IRF) was $180 \mathrm{fs}$. Transient spectra were averaged for $5 \mathrm{~s}$. Kinetic traces corresponding to the ground state bleach and excited state absorption for each spectrum were fit using a Levenberg-Marquardt nonlinear least squares fit to a sum of exponentials convoluted with a Gaussian instrument response function. 


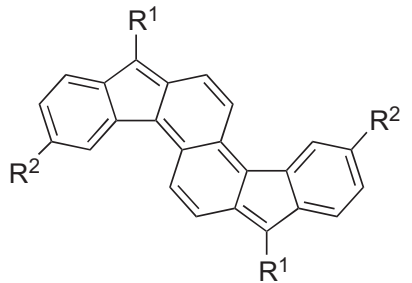

1a, $R^{1}=$ mesityl, $R^{2}=$ tert-butyl 1b, $R^{1}=R^{2}=H$

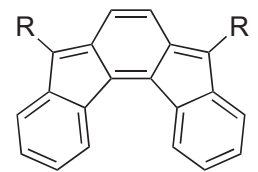

3a, R = (triisopropylsilyl)ethynyl 3b, $\mathrm{R}=\mathrm{H}$

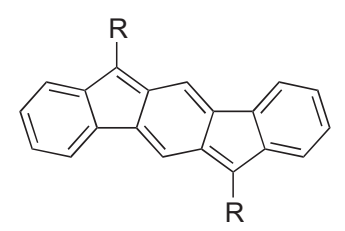

2a, R = (triisopropylsilyl)ethynyl 2b, $\mathrm{R}=\mathrm{H}$

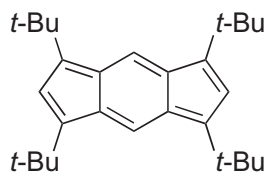

4
Figure 1. Structures of indenofluorene and fluorenofluorene derivatives investigated in this letter (1-3) as well as the structure of a related indenofluorene subunit, $s$-indacene (4).

\subsection{Computational methods}

Geometries were optimized using the fully optimized reaction space (FORS) method within the General Atomic and Molecular Electronic Structure System (GAMESS) package of programs [25]. This method can formally account for the static electronic correlation present in these systems. Further approximations are still needed to make this a manageable problem with presently available computational resources, so the pendant aryl/ethynyl groups were omitted and only a minimal active space was used-four electrons in four orbitals, denoted FORS $(4,4)$. Single point energies were also computed using multiconfigurational quasidegenarate perturbation theory (MCQDPT2) [26]. The cc-pVDZ basis set [27] was used for all cases, except for TD-DFT where the 6-311G(2df,2p) basis set [28] was used; thus, all electronic ground state structures were minimized using FORS $(4,4) / c c-p V D Z$. In the case of the photoexcited states, state averaging was done with equal weights given to the $S_{0}$ and $S_{1}$ states, denoted SA-FORS $(4,4)$. The $S_{0}$ and $S_{1}$ geometries were verified as stationary points by frequency analysis. No symmetry was enforced when searching for the conical intersections and the geometries were considered minimized when a maximum gradient of less than 0.0004 Hartree/bohr was located, as set by OPTTOL in GAMESS. Single point TD-DFT calculations were carried out using CAM-B3LYP [29,30]. Cartesian coordinates for structures are available as supplementary files named according to the scheme in text. The programs Macmolplt [31], Chemcraft [32], and CYLview [33] were used to generate and view structures. Considering the approximations used, the results obtained are only considered qualitatively correct as a larger active space, basis set and dynamic electron correlation would be needed for quantitatively correct results, but are beyond the scope and computational expense of this current study.

\section{Results and discussion}

The first system explored using transient absorption spectroscopy in solution was fluoreno[4,3-c]fluorene derivative 1a [10]. The initial absorptive feature that appears at approximately $675-800 \mathrm{~nm}$ (Figure 2a) is likely related to the $S_{2}$ state, as the calculated $S_{0}-S_{1}$ transition is forbidden in this system (vide infra). Rapid internal conversion in less than a picosecond resulted in a blue-shifted absorptive feature corresponding to $S_{1}$, which significantly overlaps with the ground state bleach from $470 \mathrm{~nm}$ to $650 \mathrm{~nm}$. This lowest-lying excited state then decays with the ultrashort lifetime of $\tau=11.9 \pm 3.5 \mathrm{ps}$ (Figure $2 \mathrm{~b}$ ). Because the internal conversion from $S_{2}$ to $S_{1}$ occurs so quickly, it is difficult to resolve the spectral signature from the intrinsic chirp from the probe pulse, even utilizing global analyses like singular value decomposition (SVD). SVD is able to spectrally resolve a shift in the excited state from redder to bluer wavelengths that is associated with vibrational cooling processes (Figure S2); however, the rate of internal conversion from $S_{1}$ to $S_{0}$ is fast enough to be competitive with vibrational cooling, which complicates assignment of individual processes rates in single-wavelength kinetic fits where the cooling can be observed (i.e., red of the $S_{1}$ absorption maximum).

We also studied the related but smaller derivatives indeno[1,2b]fluorene (2a) [13] and indeno[2,1-c]fluorene (3a) [11]. Compound 2a also exhibited sub-picosecond internal conversion from the $S_{2}$ state, that absorbs from $580 \mathrm{~nm}$ to $700 \mathrm{~nm}$, to the $S_{1}$ state, followed by excited state decay (Figure 2c). Once again, the lower excited state occupies a similar spectral region as the ground state bleach, from approximately $500 \mathrm{~nm}$ to $575 \mathrm{~nm}$. The $S_{1}$ excited state lifetime of 2a was $\tau=9.7 \pm 0.9$ ps (Figure $2 \mathrm{~d}$ ). Vibrational cooling also takes place in $\mathbf{2 a}$, but again the short lifetimes of all the relaxation processes make it difficult to assign a rate for this process.

In 3a, unlike 1a and 2a, the $S_{0}-S_{1}$ transition is allowed, and thus there is some population in both $S_{2}$ and $S_{1}$ upon initial excitation at this wavelength. In addition to sub-picosecond internal conversion, a slower blue-shift in the region of 500-600 nm can be assigned a lifetime of $\tau=1.8 \mathrm{ps}$ (Figure 2e), which corresponds well to rates of intramolecular vibrational cooling to the lowest excited state observed in many systems [34-36]. This shift was followed by excited state decay in $\tau=9.5 \pm 1.7 \mathrm{ps}$ (Figure $2 \mathrm{f}$ ). Thus, the same $S_{1}$ decay behavior was observed in solutions of $\mathbf{1 a}, \mathbf{2 a}$ and 3a, indicating picosecond excited state decay is common among these derivatives containing de-aromatized quinoidal cores. The related substructure of indenofluorene, $s$-indacene derivative $\mathbf{4}$, was found to have lifetimes of $\tau=18 \mathrm{ps}$ and $\tau=2.5 \mathrm{ps}$ for the $S_{1}$ and $S_{2}$ states, respectively. The lifetimes of 4 are similar to excited states for the larger systems of this study, indicating a similar relaxation mechanism may be responsible [37-39]. The extremely short ca. 10-20 ps excited state lifetimes explain why 1-4 are non-emissive molecules, since fluorescence is not a competitive process at this time scale [40].

We wanted to understand how rapid internal conversion of the excited state to the ground state takes place; thus, we took inspiration from the studies of 4 . It is believed $\mathbf{4}$ is able to efficiently return to the ground state through a potential energy surface crossing between $S_{1}$ and $S_{0}$ states [21]. We therefore searched computationally for a conical intersection between the $S_{1}$ and $S_{0}$ states of 1-3.

Interestingly, TD-DFT calculations using CAM-B3LYP predict a symmetry forbidden $S_{0} \rightarrow S_{1}$ transition for $\mathbf{1 b}$ and $\mathbf{2 b}$, the compounds containing an inversion center, which is attributed to a HOMO- $1 \rightarrow$ LUMO transition; however, the $S_{0} \rightarrow S_{1}$ transition is allowed for $\mathbf{3 b}$, which does not possess an inversion center (see Supporting Information). The smallest active space for exploring the conical intersection was therefore deemed to consist of 4 electrons and 4 orbitals to include the HOMO- 1 . The FORS $(4,4)$ results for $\mathbf{1 b}, \mathbf{2} \mathbf{b}$, and $\mathbf{3 b}$ show that the $S_{1}$ state differs from the ground state in that the localized double bond character of the central rings becomes attenuated. Here it should be noted that FORS overestimates the energy of the $S_{0} \rightarrow S_{1}$ transition and the $S_{0} \rightarrow S_{1}$ reorganization energy; for example in 3a the measured $S_{0} \rightarrow S_{1}$ energy is $1.48 \mathrm{eV}$ while that predicted by FORS for $\mathbf{3 b}$ is $3.29 \mathrm{eV}$. This can be partially remedied by the inclusion of dynamic correlation 


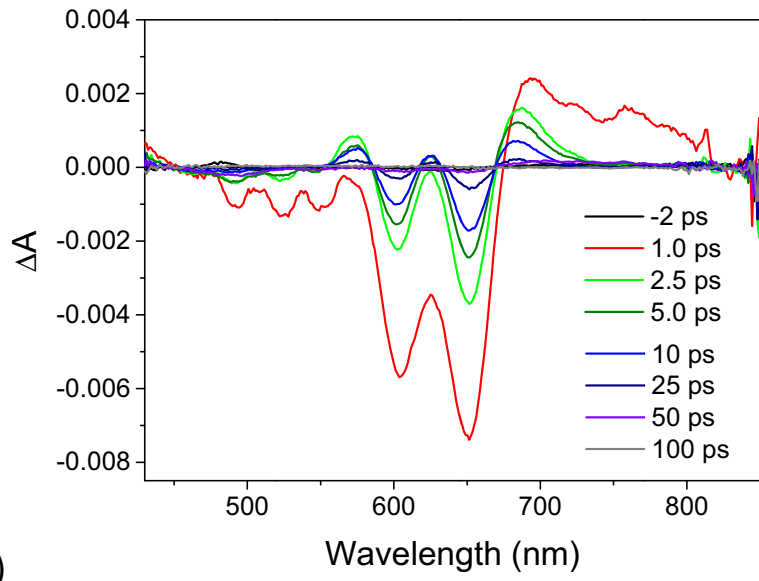

(a)

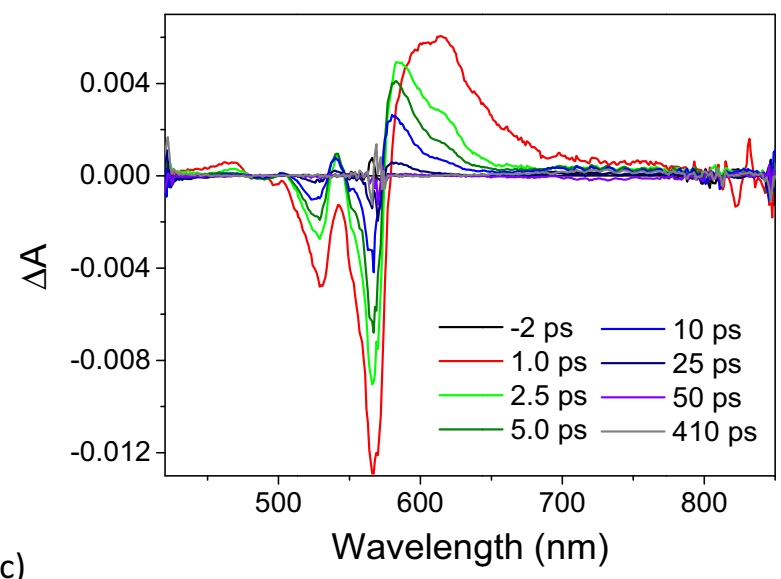

(c)

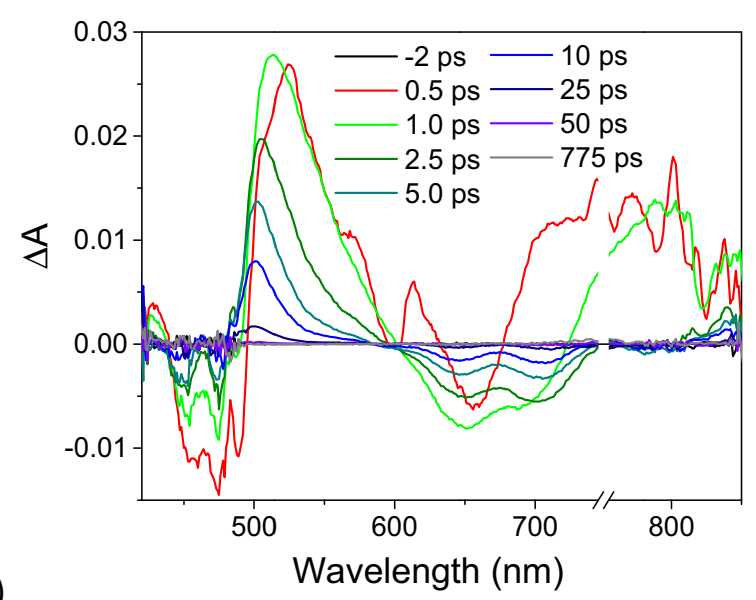

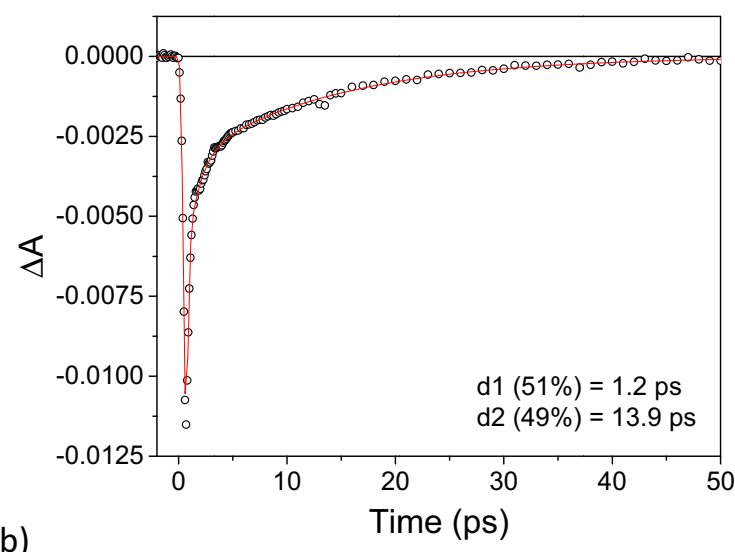

(b)

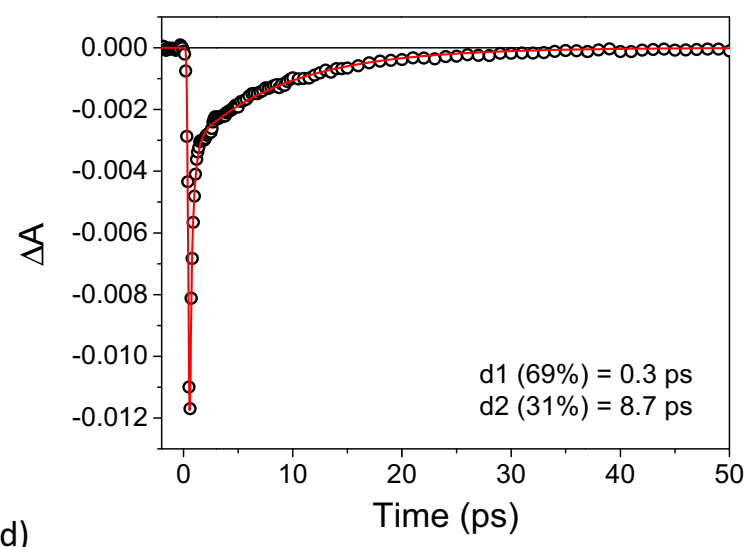

(d)

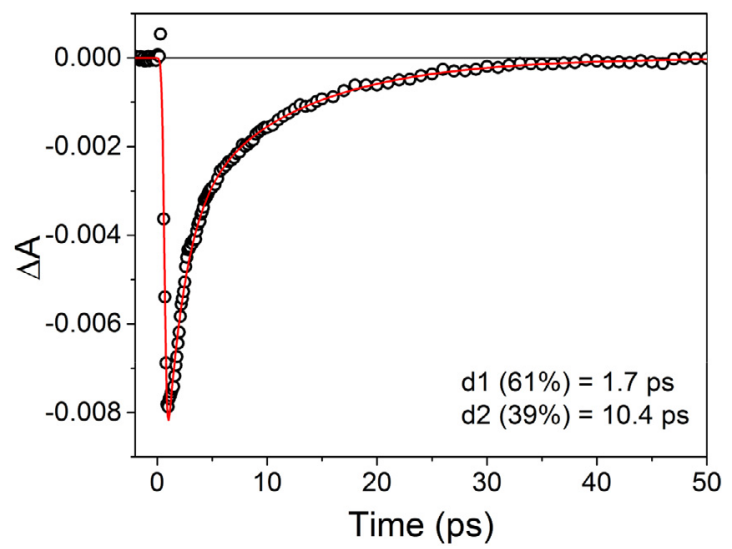

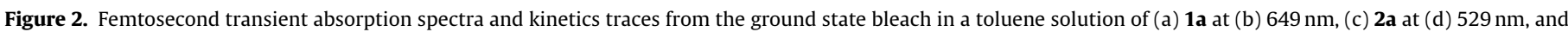
(e) 3a at (f) $645 \mathrm{~nm}$, showing recovery corresponding to rapid excited state decay pathways.

using MCQDPT2. A SA-MCQDPT2(4,4) single point energy calculation predicts the $\mathbf{3 b}$ vertical $S_{0} \rightarrow S_{1}$ transition to be $2.27 \mathrm{eV}$ (546 $\mathrm{nm})$, closer in alignment with experimental data for $3 \mathbf{a}(1.93 \mathrm{eV}, 647$ $\mathrm{nm})$. The SA-MCQDPT2 $S_{0} \rightarrow S_{1}$ reorganization energies are $0.70 \mathrm{eV}$ for $\mathbf{1 b}, 0.68 \mathrm{eV}$ for $\mathbf{2 b}$, and $0.14 \mathrm{eV}$ for $\mathbf{3 b}$. Continuing on from the $S_{1}$ geometry, only a small perturbation is required to reach the geometry of the conical intersection (Figure 3 ). This facile $S_{1}$-toconical intersection rearrangement is reflected in the geometries as well as the potential energy surfaces. The energy difference for the $S_{1}$ potential energy surface going from the $S_{1}$ geometry to the conical intersection is $0.03 \mathrm{eV}$ for $\mathbf{1 b}, 0.26 \mathrm{eV}$ for $\mathbf{2 b}$, and $0.05 \mathrm{eV}$ for $\mathbf{3 b}$. While qualitative in nature, the small difference in energy and geometry between the $S_{1}$ and conical intersection geometry strongly implicates that a conical intersection is the likely mechanism for efficient non-radiative decay to the ground state, similar to 4 . 


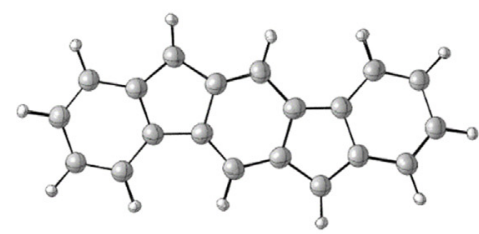

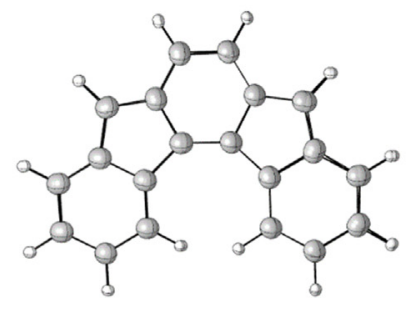

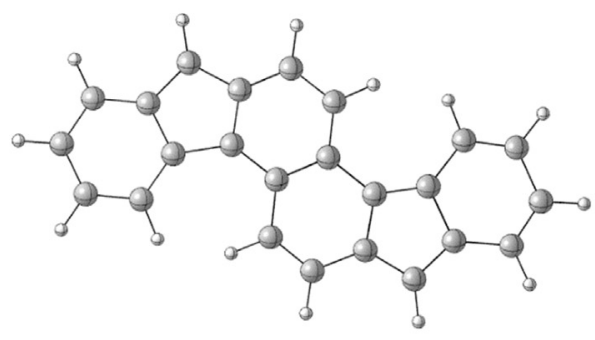
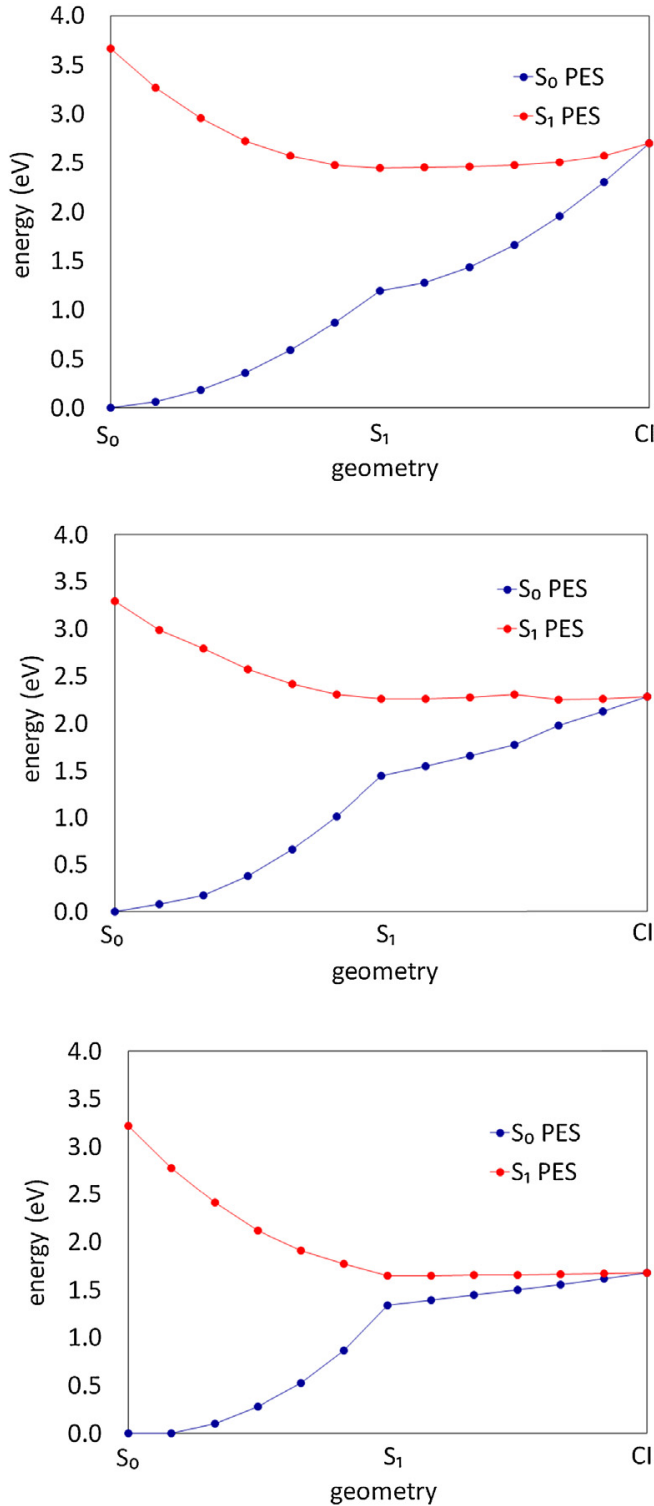

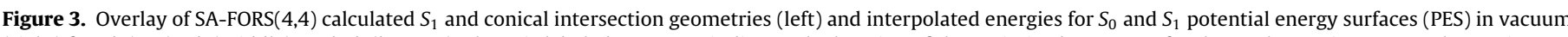

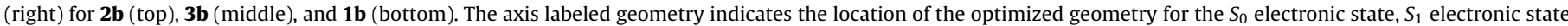
and conical intersection (CI) in the plot. Five linearly interpolated geometries between the $S_{0}$ and $S_{1}$ as well as the $S_{1}$ and $\mathrm{CI}$ are included on the plot.

\section{Conclusions}

We have reported the solution excited state lifetimes for quinoidal molecules 1a-3a as measured by transient absorption spectroscopy, and computationally explored the $S_{1} / S_{0}$ potential energy surface crossing for simplified models systems $\mathbf{1 b}-\mathbf{3 b}$. This series of quinoidal molecules was found to have very short $S_{1}$ excited state lifetimes (ca. 9-12 ps) due to an easily accessible $S_{1} / S_{0}$ conical intersection. This work helps lay a foundation for future explorations on solid thin films of these molecules and other related pi-conjugated quinoidal compounds.

\section{Acknowledgments}

This work was supported by the National Science Foundation (CHE-1301485, M.M.H.) and the Chemical Sciences, Geosciences, and Biosciences Division, Office of Basic Energy Sciences, DOE (DE-FG02-99ER14999, M.R.W.). B.D.R. acknowledges the NSF for a GK-12 fellowship (DGE-0742540). We thank Dr. Johannes
Gierschner for valuable initial discussions, Samuel Eaton, Eric Margulies, and Dr. Ryan Young for constructive discussions, and Aaron Fix for supplying compound $\mathbf{3 a}$.

\section{Appendix A. Supplementary data}

Supplementary data associated with this article can be found, in the online version, at doi:10.1016/j.cplett.2014.10.031.

\section{References}

[1] S.-S. Sun, L.R. Dalton (Eds.), Introduction to Organic Electronic and Optoelectronic Materials and Devices, CRC Press, Boca Raton, FL, 2008.

[2] S.R. Forrest, Nature 428 (2004) 911

[3] E. Wang, W. Mammo, M.R. Andersson, Adv. Mater. 26 (2014) 1801.

[4] C.D. Dimitrakopoulos, P.R.L. Malenfant, Adv. Mater. 14 (2002) 99.

[5] M. Bendikov, F. Wudl, D.F. Perepichka, Chem. Rev. 104 (2004) 4891

[6] J. Huang, J.-H. Su, H. Tian, J. Mater. Chem. 22 (2012) 10977.

[7] D. Chen, et al., Org. Electron. 15 (2014) 1197.

[8] S. Karak, V.S. Reddy, S.K. Ray, A. Dhar, Org. Electron. 10 (2009) 1006.

[9] M. Heeney, et al., Macromolecules 37 (2004) 5250.

[10] B.D. Rose, C.L. Vonnegut, L.N. Zakharov, M.M. Haley, Org. Lett. 14 (2012) 2426 
[11] A.G. Fix, P.E. Deal, C.L. Vonnegut, B.D. Rose, L.N. Zakharov, M.M. Haley, Org. Lett. 15 (2013) 1362

[12] D.T. Chase, et al., J. Am. Chem. Soc. 134 (2012) 10349.

[13] D.T. Chase, et al., Angew. Chem. Int. Ed. 50 (2011) 11103.

[14] B.D. Rose, et al., J. Am. Chem. Soc. 136 (2014) 9181.

[15] A. Shimizu, Y. Tobe, Angew. Chem. Int. Ed. 50 (2011) 6906.

16] M.J. Kendrick, et al., J. Phys. Chem. C 116 (2012) 18108.

[17] J. Nishida, S. Tsukaguchi, Y. Yamashita, Chem. Eur. J. 18 (2012) 8964.

[18] S. Ito, T. Minami, M. Nakano, J. Phys. Chem. C 116 (2012) 19729.

19] S.W. Eaton, et al., J. Am. Chem. Soc. 135 (2013) 14701.

[20] M.C. Hanna, A.J. Nozik, J. Appl. Phys. 100 (2006) 074510/1.

[21] M.J. Bearpark, P. Celani, F. Jolibois, M. Olivucci, M.A. Robb, F. Bernardi, Mol. Phys 96 (1999) 645.

[22] R.F. Kelley, R.H. Goldsmith, M.R. Wasielewski, J. Am. Chem. Soc. 129 (2007) 6384.

[23] J.E. Bullock, et al., J. Phys. Chem. B 114 (2010) 1794.

[24] A.S. Lukas, S.E. Miller, M.R. Wasielewski, J. Phys. Chem. B 104 (2000) 931.

[25] M.W. Schmidt, et al., J. Comput. Chem. 14 (1993) 1347.

[26] H. Nakano, J. Chem. Phys. 99 (1993) 7983.
[27] T.H. Dunning Jr., J. Chem. Phys. 90 (1989) 1007.

[28] R. Krishnan, J.S. Binkley, R. Seeger, J.A. Pople, J. Chem. Phys. 72 (1980) 650

[29] T. Yanai, D.P. Tew, N.C. Handy, Chem. Phys. Lett. 393 (2004) 51.

[30] Y. Tawada, T. Tsuneda, S. Yanagisawa, T. Yanai, K. Hirao, J. Chem. Phys. 120 (2004) 8425

[31] B.M. Bode, M.S. Gordon, J. Mol. Graph. Model. 16 (1998) 133.

[32] G. Zhurko, Chemcraft, http://www.chemcraftprog.com

[33] C. Legault, CYLview, Université de Sherbrooke, 2009 http://www.cylview.org

[34] A. Pigliucci, G. Duvanel, L.M.L. Daku, E. Vauthey, J. Phys. Chem. A 111 (2007) 6135.

[35] D. Schwarzer, C. Hanisch, P. Kutne, J. Troe, J. Phys. Chem. A 106 (2002) 8019

[36] T. Elsaesser, W. Kaiser, Annu. Rev. Phys. Chem. 42 (1991) 83.

[37] R. Klann, R.J. Bäuerle, F. Laermer, T. Elsaesser, M. Niemeyer, W. Lüttke, Chem. Phys. Lett. 169 (1990) 172

[38] C. Gellini, P.R. Salvi, K. Hafner, J. Phys. Chem. 97 (1993) 8152

[39] C. Gellini, L. Angeloni, P.R. Salvi, G. Marconi, J. Phys. Chem. 99 (1995) 85.

[40] N. Turro, V. Ramamurthy, J. Scaiano, Modern Molecular Photochemistry of Organic Molecules, University Science Books, Sausalito, CA, 2010. 